\title{
Aprendizaje colaborativo en odontología conservadora mediante el uso de la lluvia de ideas como recurso educativo
}

\section{Collaborative learning in conservative dentistry by using the brainstorming approach}

\author{
Erika Melissa Gutiérrez ${ }^{1}$, Diego Salmerón ${ }^{2}$, Antonia Alonso ${ }^{3}$, Nicanor Morales- \\ Delgado $^{4^{*}}$
}

1 Departamento de Anatomía Humana y Psicobiología. Facultad de Medicina. Universidad de Murcia (Murcia) erikamelissa.gutierrezc@um.es. ORCID: https://orcid.org/0000-0001-98412238

2 Departamento de Ciencias Sociosanitarias, Universidad de Murcia, Murcia. CIBER Epidemiología y Salud Pública (CIBERESP), Murcia. IMIB-Arrixaca, Murcia dsm@um.es. ORCID: https://orcid.org/0000-0002-4574-6880

3 Departamento de Anatomía Humana y Psicobiología. Facultad de Medicina. Universidad de Murcia (Murcia) antoniaaf@um.es. ORCID: https://orcid.org/0000-0002-8214-8054

4 Departamento de Histología y Anatomía. Facultad de Medicina. Universidad Miguel Hernández (Alicante) nmorales@umh.es. ORCID: https://orcid.org/0000-0002-6716-9041

* Correspondencia: nmorales@umh.es

Recibido: 5/11/2020; Aceptado: 27/11/2020; Publicado: 27/11/2020

Resumen: La implementación del Aprendizaje Colaborativo (AC) ha aumentado notablemente en los últimos años, ya que permite la construcción del pensamiento, crear clases más dinámicas y el desarrollo de habilidades sociales y comunicativas de los estudiantes. Estas últimas son primordiales para el desempeño profesional en áreas sanitarias donde el trabajo colaborativo es fundamental. Nuestro estudio descriptivo de corte transversal evaluó el efecto de la implementación del AC en dos entornos de aprendizaje distintos, tradicional y digital, en el módulo de Técnicas de Ayuda Odontológica/Estomatológica. La población de estudio $(\mathrm{n}=25)$ fue dividida aleatoriamente en 8 grupos de 3 o 4 estudiantes. Como herramientas digitales se utilizaron Stormboard y Socrative para realizar la lluvia de ideas y cuestionarios de repaso, respectivamente. Después de la impartición de 6 sesiones teóricas y familiarización con el uso de las plataformas digitales, se realizaron dos actividades colaborativas (instrumental y caso clínico) de manera tradicional y digital. Al término de cada actividad, se lanzaron cuestionarios gamificados Space Race individuales para valorar los conocimientos adquiridos. Finalmente, se aplicó un examen teórico final y una encuesta anónima para valorar la metodología aplicada. Nuestros resultados reflejan que la adquisición de contenidos en un entorno colaborativo tradicional y virtual es bastante similar. Además, un alto porcentaje del alumnado consideró que el empleo de las herramientas digitales les ayudó a ampliar conocimientos sobre el tema estudiado y en su proceso de aprendizaje. En conjunto, Stormboard es una herramienta digital colaborativa innovadora que se podría aplicar a otros Ciclos Formativos o niveles educativos superiores como el universitario, con las adaptaciones y mejoras pertinentes.

Palabras clave: Aprendizaje colaborativo; Formación Profesional; Stormboard; lluvia de ideas; Socrative.

Abstract: The implementation of Collaborative Learning has increased appreciably in recent years since it facilitates learning, creates more dynamic classes and promotes students' social and communication skills. The latter is crucial for healthcare workers, where collaborative work plays a pivotal role. Our cross- 
sectional descriptive study aimed to evaluate the effect of collaborative learning implementation in two different learning environments, traditional and digital, in the module of Techniques of Odontostomatology. It was conducted on a sample of 25 students, which were randomly divided into eight groups with 3-4 people per group. Stormboard and Socrative were used as digital tools for brainstorming and quiz activities, respectively. After the theoretical sessions, collaborative activities were carried out in different environments (traditional and digital). At the end of each activity, individual Socrative Space Race questionnaires were performed. Finally, a theoretical exam and an anonymous questionnaire were applied to assess the knowledge gained and the perception of students on this teaching strategy. Our results suggest that the acquisition of contents in a traditional and virtual collaborative environment is quite similar. Moreover, a high percentage of the students considered that digital tools helped them expand their knowledge of the subject studied and their learning process. Altogether, these observations point out that Stormboard can be an innovative and collaborative digital tool, applicable to Professional Cycles or university degree programs, with appropriate adaptations and improvements.

Keywords: Collaborative learning; Vocational Training; Stormboard; brainstorming; Socrative.

\section{Introducción.}

El enfoque e impartición de la Educación Sanitaria (ES) en el aula prácticamente no ha variado en las últimas décadas. La enseñanza continúa siendo eminentemente tradicional. El profesor de manera unidireccional comparte sus conocimientos con los discentes mediante una clase magistral y ellos los aprenden y/o memorizan de forma individual. Sin embargo, en las últimas décadas, todo nuestro entorno ha experimentado cambios importantes, especialmente a nivel social y tecnológico, brindándonos así múltiples opciones para desarrollar el proceso de enseñanzaaprendizaje de forma aún más interactiva, con nuevos recursos didácticos para que nuestro alumnado aprenda y trabaje cada vez mejor. Además, el trabajo virtual ha incrementado notablemente a lo largo de los últimos meses como consecuencia de la pandemia COVID-19, redundando, a su vez, y a diferencia de lo que sucedía con anterioridad, en el trabajo remoto con equipos que se encuentran en diferentes espacios territoriales. Por tal motivo, existe una gran necesidad de formar a nuestros estudiantes en todas las herramientas básicas necesarias, que fomenten habilidades sociales y digitales ajustadas a la nueva realidad, con tal de preservar el bienestar individual y el funcionamiento organizacional óptimo (1).

En el contexto social actual, es necesario crear e implementar diferentes metodologías en la ES que nos permitan desarrollar valores en nuestros alumnos como la responsabilidad, la comunicación productiva y el trabajo en equipo, además de las necesarias habilidades sociales, que cobran especial relevancia dentro de las dinámicas grupales, como la tolerancia, la empatía y la capacidad de escucha, entre otras (2). Todas ellas son indispensables para fomentar un buen trato y una adecuada atención de los pacientes, pero también contribuyen a potenciar una percepción más apropiada de lo que significa el trabajo en equipo, promoviendo la integración oportuna de los futuros profesionales sanitarios en equipos multidisciplinares, en los que se comparten los conocimientos para conseguir un bien común.

La ES del siglo XXI debe responder a los retos que plantea la sociedad de la información, la reestructuración productiva y la sociedad del conocimiento, ya que el 
actual proceso de informatización y digitalización general del mundo puede ser contemplado como la culminación de otro que cambiará radicalmente la percepción del tiempo y del espacio, formando la base cultural de las grandes transformaciones económicas (3). Dicho contexto en el que se integrarán los estudiantes del futuro debe promover una formación enfocada no sólo a conocimientos fisiológicos o morfológicos, sino además humanísticos, sociales, metodológicos, científicos, filosóficos y éticos, cuyos esfuerzos deben ir dirigidos a la consecución de lo que conocemos como relación de ayuda (4). Por tanto, la ES actual debe adaptar las metodologías y herramientas docentes en la Era Digital en la que nos encontramos inmersos para conseguir una mayor motivación del alumnado y, por lo tanto, mejores resultados (5).

Una metodología didáctica que resulta ser útil para poner en práctica las premisas anteriores en el alumnado es la que promueve el aprendizaje colaborativo (AC). En esta aproximación metodológica, los alumnos, que trabajan de forma conjunta, ordenada y coordinada en el aula, ejercen un papel activo, creativo y autónomo en su propio aprendizaje, construyendo su conocimiento a partir de sus experiencias previas y del entorno en el que se encuentran (6).

Por otro lado, la formación online, gracias al apoyo y uso creciente de las nuevas Tecnologías de la Información y la Comunicación (TIC), ha abierto un nuevo y amplio abanico de posibilidades en la que se puede desarrollar un entorno híbrido para trabajar tanto a distancia como de forma presencial. La evidencia acumulada indica que las TIC, siempre y cuando se utilicen de forma adecuada, con un fin didáctico claro, pueden favorecer y potenciar el aprendizaje y la colaboración entre los alumnos, repercutiendo positivamente a su vez en su carrera profesional (7). Algunas ventajas que las TIC proporcionan tanto al docente como a los estudiantes son: la motivación, el interés, la interacción de la información, el trabajo cooperativo y la retroalimentación (8). Son herramientas que, gracias a su versatilidad, ayudan a los docentes y estudiantes a adaptarse a los cambios contemporáneos, adecuando la enseñanza a nuevos modelos que sean más funcionales y productivos. Lo que el alumno aprende en un entorno virtual no es simplemente una copia o una reproducción de lo que en ese entorno se le presenta como contenido a aprender, sino una reelaboración de ese contenido mediada por la estructura cognitiva del aprendiz (9).

Este nuevo paradigma socioeducativo nos ha llevado a diseñar una nueva estrategia metodológica para la enseñanza en ES, integrando el AC con el uso de las TIC, que se pueden ejecutar fácilmente desde un dispositivo móvil (tableta, smartphone, etc), con el fin de desarrollar clases más atractivas y dinámicas para los alumnos, manteniéndolos más motivados, contribuyendo así a reforzar y fijar, de forma conjunta y coordinada, los conceptos e ideas impartidos. En concreto, el objetivo principal del presente estudio consistió en evaluar el efecto de la implementación del AC en dos entornos de aprendizaje distintos, tradicional y digital, en la Unidad de Trabajo "Instrumental, materiales y procedimientos clínicos en Odontología conservadora", perteneciente al módulo profesional de Técnicas de Ayuda Odontológica/Estomatológica, mediante el uso de la plataforma multimedia colaborativa Stormboard (https://stormboard.com).

\section{Métodos.}

\subsection{Participantes y diseño}

El presente estudio, descriptivo y de corte transversal, se desarrolló durante el curso académico 2019-2020, específicamente en el alumnado matriculado en el 
módulo profesional de Técnicas de Ayuda Odontológica/Estomatológica del Ciclo de Grado Medio de Formación Profesional “Cuidados Auxiliares de Enfermería” (I.E.S. Los Albares, Murcia).

La muestra poblacional estuvo constituida por 23 estudiantes, mayoritariamente mujeres (85\%), con un promedio de edad de 21 años y con estudios completados de 4 o ESO (61\%). Ésta se distribuyó de forma aleatoria en dos grupos con la finalidad de obtener grupos heterogéneos para las actividades de aprendizaje colaborativo propuestas. El grupo control $(\mathrm{n}=12)$ realizó las actividades mediante la metodología tradicional (fichas con cartulinas; Figura 1A), mientras que el grupo experimental, de intervención o de innovación $(\mathrm{n}=11)$ trabajó con la herramienta digital Stormboard (Figura 1B). No obstante, se registró cierto absentismo puntual durante el transcurso de este estudio (que abarcó 5 semanas), por lo que solo se ha tenido en cuenta la participación real en el cálculo estadístico para cada actividad colaborativa y gamificada propuesta (Tabla 1). Dentro de cada grupo poblacional, los estudiantes trabajaron en equipos de 3 o 4 personas ( 4 grupos por cada modalidad).
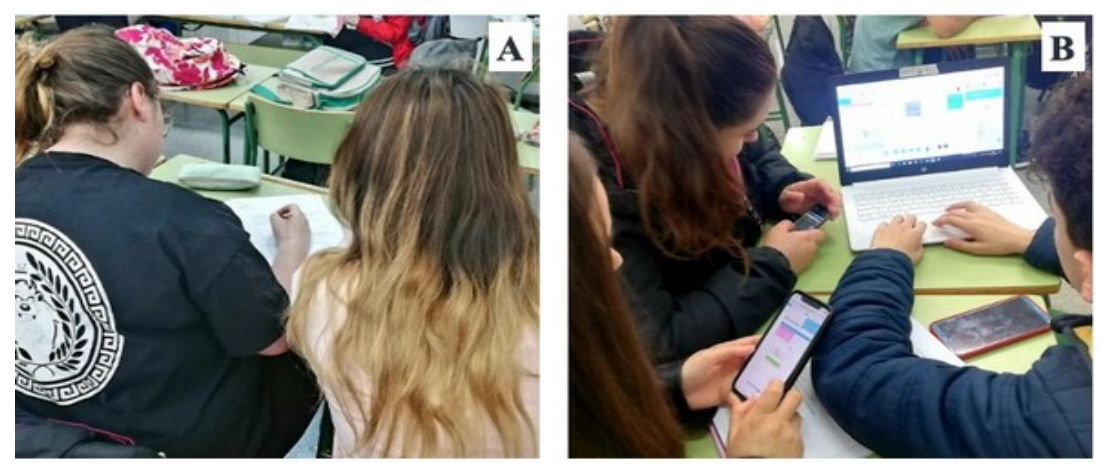

Figura 1. Grupos control (A) y experimental (B) durante el desarrollo de las actividades prácticas.

\subsection{Temporalización}

Para la realización de este trabajo, se llevaron a cabo dos estrategias de aprendizaje colaborativo distintas (instrumental y casos clínicos) en las clases prácticas de la Unidad de Trabajo (UT) número 6 (UT 6). Tal y como se muestra en la Figura 2, primero se impartieron 6 sesiones teóricas con la ayuda de PowerPoint. Cada clase magistral abarcó una duración máxima de 55 minutos. Posteriormente, se llevaron a cabo dos sesiones o actividades de aprendizaje colaborativo independientes (lluvia de ideas), que tuvieron por objeto fomentar el aprendizaje en equipo y potenciar la capacidad de trabajo a nivel individual y grupal. Ambas competencias son fundamentales en el Área Sanitaria, permitiéndoles intervenir activamente en procesos de decisión compartida, de forma positiva y creativa, desarrollando su espíritu crítico constructivo y aportando soluciones centradas en un objetivo común (10).

La primera práctica desarrollada en el aula estuvo centrada en el manejo y conocimiento del instrumental de uso dental. Cada equipo del grupo control (método tradicional), para completar esta actividad de AC, tuvo que rellenar una ficha con la ayuda de tres cartulinas (pistas), que fueron distribuidas por distintos lugares del aula. Los alumnos contaron con un libro de texto de referencia en Odontología como apoyo adicional para la resolución de la actividad práctica planteada (11). 


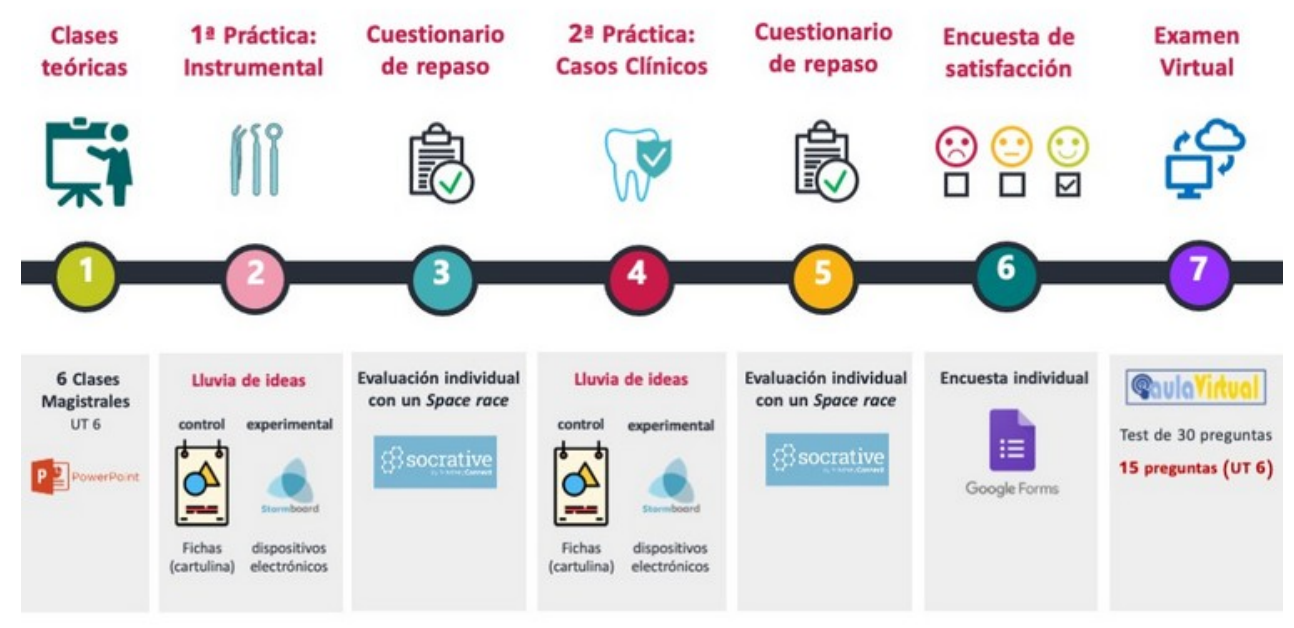

Figura 2. Esquema cronológico de las actividades colaborativas y gamificadas que se realizaron en este estudio.

Por otro lado, el grupo experimental (metodología basada en las TIC) utilizó la aplicación Stormboard para realizar un cuadro sinóptico sobre dicho material odontológico. Se creó una lluvia de ideas (storm) con un identificador (identification o ID) y una contraseña específica para cada equipo. Algunos equipos realizaron la actividad de brainstorming desde sus propios smartphones o tabletas a través de la aplicación móvil Stormboard, mientras que otros la hicieron desde los ordenadores disponibles en el aula a través de la propia página web de esta herramienta online. Durante el desarrollo de la actividad, los alumnos buscaron y aprendieron el nombre, descripción y uso de cada uno de los instrumentos dentales. Todos los miembros de un mismo equipo, de forma simultánea, agregaron los textos e imágenes que consideraron oportunos para completar la plantilla proporcionada por la profesora a través de sus dispositivos. En la última parte de esta actividad práctica, ambos grupos (control y experimental) manipularon el material instrumental, reforzando así lo que habían aprendido previamente.

La segunda práctica estuvo centrada en la resolución de casos clínicos, los cuales relacionaron los conocimientos teóricos previos impartidos con los aprendidos en la práctica anterior sobre instrumental dental. En concreto, los equipos del grupo control y experimental tuvieron que resolver una serie de preguntas relacionadas con el diagnóstico y clasificación del caso clínico, así como los materiales e instrumental dental que necesitarían preparar a un odontólogo para atender debidamente al paciente según el planteamiento dado. De modo similar a la práctica anterior, la estrategia empleada en la resolución de las cuestiones planteadas sobre los casos clínicos fue diferente para cada grupo. El grupo control volvió a contar con la ayuda de las fichas creadas por la profesora y el libro de apoyo (11), mientras que el equipo experimental utilizó únicamente la aplicación Stormboard. Al término de cada práctica, se lanzó un cuestionario gamificador de carrera espacial (o Space race) con el objetivo de repasar y asentar los conocimientos adquiridos. En ambas ocasiones, tanto los alumnos del grupo control como experimental respondieron los cuestionarios, de forma individual, a través de la aplicación móvil Socrative Student (https://b.socrative.com/login/student/). 
Por otra parte, para conocer la opinión de los alumnos tanto de la metodología aplicada como de la actuación docente, se les solicitó que cumplimentaran, de forma voluntaria y anónima, una encuesta. Esta constó de 27 preguntas y fue creada con la herramienta online gratuita Google Forms. Las preguntas del cuestionario se basaron en una encuesta previamente validada (12). Para los dos bloques, metodología aplicada y actuación docente, se utilizaron escalas Likert con cinco opciones de respuesta, para indicar su grado de acuerdo o desacuerdo con cada una de las afirmaciones, siendo 1 «totalmente en desacuerdo» y 5 «totalmente de acuerdo». La encuesta fue formulada considerando 3 dimensiones: a) grado de satisfacción de los estudiantes sobre las herramientas digitales Stormboard y Socrative; b) grado de satisfacción de los estudiantes del grupo control sobre la metodología tradicional empleada; y c) grado de satisfacción sobre la actuación docente (datos no mostrados).

Por último, debido a la entrada en vigor del Estado de Alarma por la crisis sanitaria de la COVID-19 Real Decreto 463/2020, de 14 de marzo, por el que se declara el estado de alarma para la gestión de la situación de crisis sanitaria ocasionada por el COVID-19 (13), para la evaluación de los conocimientos adquiridos, los alumnos realizaron un examen virtual en la plataforma educativa "Murcia Educa" (https://aulavirtual.murciaeduca.es/index.php). Los contenidos evaluados correspondieron a las siguientes UT:

- UT 4: "El diagnóstico en Odontología (I): la exploración clínica". Impartida exclusivamente por la tutora de prácticas.

- UT 5: "El diagnóstico en Odontología (II): la radiología bucal". Impartida exclusivamente por la tutora de prácticas.

- UT 6: Instrumental, materiales y procedimientos clínicos en Odontología (objeto de estudio y descrita previamente).

El examen virtual tipo test estuvo compuesto por un total de 30 preguntas de respuesta múltiple (con 4 alternativas con una sola respuesta correcta) y tuvo una duración máxima de 25 minutos. Cada 3 respuestas incorrectas restaron 0.33 puntos. La mitad del examen estuvo compuesto por preguntas de la UT6.

\subsection{Análisis estadístico}

Una vez finalizada la fase de trabajo de campo, se realizó el análisis estadístico de los resultados obtenidos mediante el software IBM SPSS (SPSS Inc., V18.0, Chicago, USA). Se calcularon los estadísticos descriptivos básicos de la muestra según grupo, los intervalos de confianza al 95\% (IC 95\%), la prueba T de Student para muestras independientes para las diferencias entre medias, y la prueba de Mann-Whitney.

\section{Resultados.}

En primer lugar, se calcularon los estadísticos descriptivos de los dos cuestionarios gamificados (carrera espacial o Space race) por grupo de interés (Tabla 1). En el primer cuestionario Socrative, el instrumental, la media de respuestas acertadas entre el grupo control y el experimental fue muy similar (16.3 vs. 16), con un rango entre 13 y 19, y 8 y 20, respectivamente (Figura 3). Sin embargo, en el cuestionario Socrative casos observamos una leve mejoría en las calificaciones, superando el $96 \%$ de los participantes la prueba. El promedio de aciertos en el grupo control fue 16, con un rango entre 6 y 20, frente a los 16.67 del grupo experimental, con un rango entre 14 y 19 (Tabla 1; Figura 3). No se observaron diferencias estadísticamente significativas entre los grupos de muestreo ( $p=0.8$ vs. $P=0.611$ ). 


\begin{tabular}{|l|c|c|c|c|c|c|c|}
\hline Instrumental & $\mathrm{N}$ & Media & DE & IC 95\% & Min. & Máx. & $\mathrm{p}$ \\
\hline Control & 10 & 16.3 & 2.00 & $14.87-17.73$ & 13 & 19 & \multirow{2}{*}{0.8} \\
\hline Experimental & 11 & 16 & 3.162 & $13.83-18.12$ & 8 & 20 & \\
\hline Casos & 12 & 16 & 3.542 & $13.75-18.25$ & 6 & 20 & \multirow{2}{*}{0.61} \\
\hline Control & 9 & 16.67 & 1.732 & $15.34-18$ & 14 & 19 & \\
\hline Experimental & 9 &
\end{tabular}

Tabla 1. Descriptivos de los cuestionarios gamificados realizados. El tamaño de la muestra $(\mathrm{N})$ es variable debido al absentismo puntual registrado. DE: desviación estándar; IC 95\% intervalo de confianza 95\%.

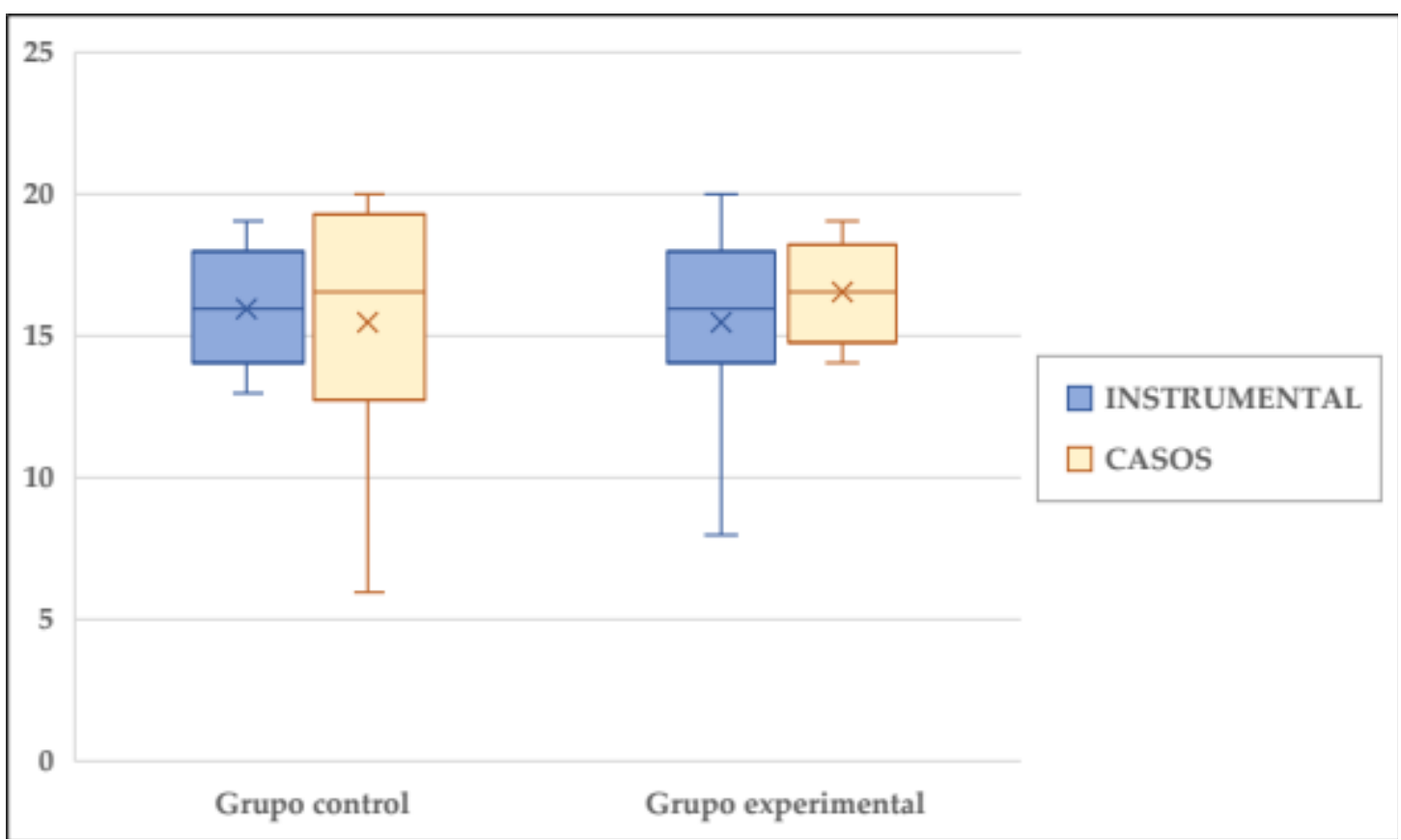

Figura 3. Diagramas de caja-bigote para la puntuación obtenida en los dos tipos de cuestionarios gamificados Socrative.

Como se ha indicado más arriba, la evaluación conjunta de las UT 4, 5 y 6 se llevó a cabo mediante un examen virtual, en el cual la mitad de las preguntas (15 cuestiones en concreto) se centraron en la UT6. Por ello, en segundo lugar, realizamos un análisis de las calificaciones individuales obtenidas tanto en la UT6 como en la nota global de dicho examen final para comprobar si se observaban diferencias de efecto entre grupos. Atendiendo a los datos de la Tabla 2, la puntuación media de respuestas correctas en la UT6 para el grupo control fue de 9.91, con un rango entre 4 y 14 , mientras que la del grupo experimental fue algo mayor, de 11.20, con un rango entre 6 y 15 (Figura 4). No se encontró que el grupo experimental tuviese resultados significativamente diferentes del control $(p=0.35)$.

En cuanto a la nota parcial del examen final, se observó que el valor promedio del grupo experimental fue mayor (7.32) al del grupo control (6.06), lo cual nos indica que el grupo que utilizó la plataforma Stormboard para el desarrollo de las actividades de 
aprendizaje colaborativo obtuvo una mejor nota parcial en el examen, aunque dichas diferencias entre ambos grupos tampoco alcanzaron significación estadística $(p=0.296$; Tabla 2).

\begin{tabular}{|c|c|c|c|c|c|c|c|}
\hline \multicolumn{8}{|c|}{ Unidad de trabajo 6 (UT6) } \\
\hline & $\mathrm{N}$ & Media & $\mathrm{DE}$ & IC 95\% & Min. & Máx. & $\mathrm{p}$ \\
\hline Control & 11 & 9.91 & 3.53 & $7.53-12.28$ & 4 & 14 & \multirow{2}{*}{0.35} \\
\hline Experimental & 10 & 11.20 & 2.49 & $9.42-12.98$ & 6 & 15 & \\
\hline \multicolumn{8}{|c|}{ Nota parcial del examen trimestral } \\
\hline Control & 11 & 6.06 & 3.16 & $3.93-8.18$ & 1.32 & 9.9 & \multirow{2}{*}{0.29} \\
\hline Experimental & 10 & 7.33 & 2.05 & $5.85-8.79$ & 3.3 & 9.9 & \\
\hline
\end{tabular}

Tabla 2. Descriptivos del número de respuestas acertadas sobre la UT6 y su correspondiente nota parcial en el examen trimestral. DE: desviación estándar; IC 95\% intervalo de confianza 95\%.

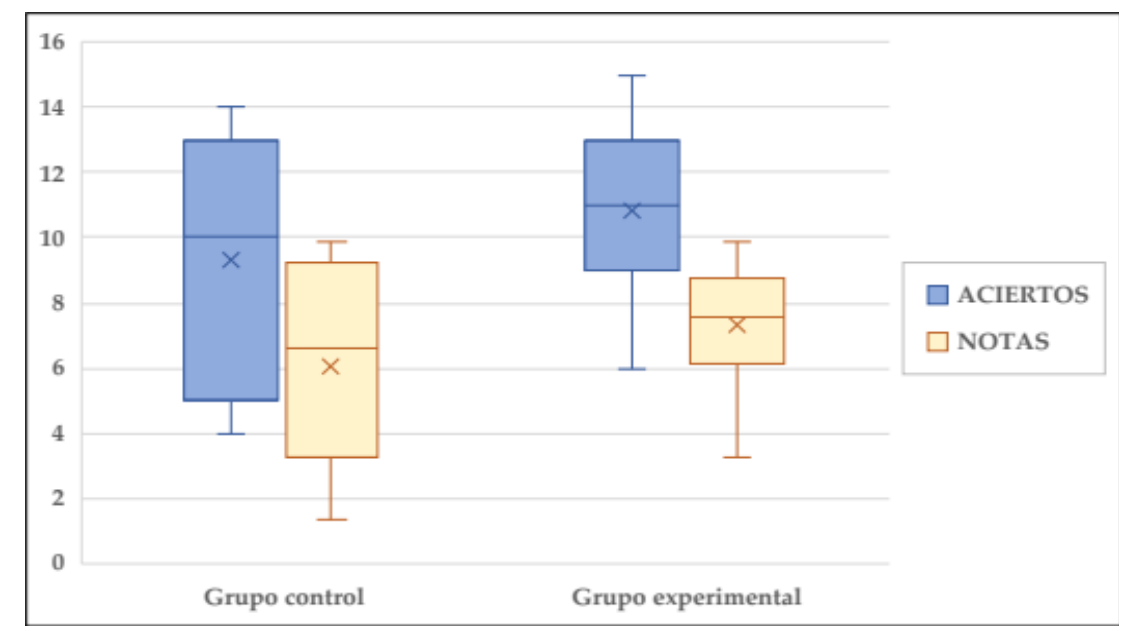

Figura 4. Diagramas de caja-bigote para la puntuación obtenida en las respuestas acertadas de la UT6 y las notas parciales.

La última parte de este estudio se centró en el análisis cuantitativo del grado de satisfacción de los estudiantes expuestos a la experiencia de innovación docente mediante una encuesta anónima y voluntaria. La mayoría de los alumnos matriculados (92\%) completó la encuesta.

En la Figura 5 se observa que la opinión general sobre el sistema de cuestionarios gamificados de Socrative es bastante favorable, a pesar de que casi la mitad del estudiantado no tenía experiencia en el uso de esta aplicación u otras similares con fines educativos. En todos los ítems se alcanzó un grado de acuerdo o muy de acuerdo superior al $80 \%$ con la pregunta pertinente, destacando una alta satisfacción de los estudiantes (del 91.5\%) con el aprendizaje móvil en el aula. Además, consideraron que el uso de este tipo de cuestionarios les ayudó a implicarse más en el desarrollo de las clases prácticas, ya que, por un lado, pudieron poner a prueba sus conocimientos sobre el tema (aproximadamente un 91\%) y, por otro lado, el uso de la aplicación a través de sus móviles mejoró su concentración y atención en clase, mostrándose más atentos y participativos (80.9\%). 


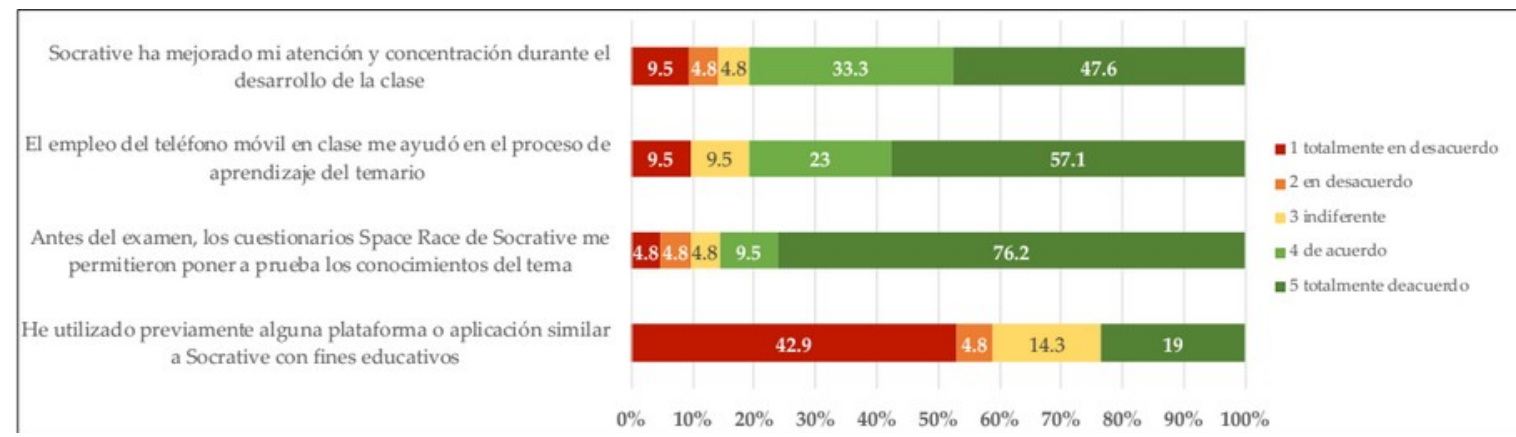

Figura 5. Valoración global de la experiencia gamificadora con los cuestionarios Socrative.

Por otro lado, los alumnos del grupo control y experimental valoraron la experiencia con las fichas de trabajo (cartulinas) y los tableros digitales (storms) de la plataforma Stormboard, respectivamente. Así, aproximadamente un $80 \%$ de los estudiantes, entre los cuales un 63,6\% jamás había usado una plataforma digital similar a Stormboard con fines educativos, manifestó sentirse satisfecho con ambas metodologías colaborativas, tradicional e innovadora, calificándolas de atractivas, productivas y útiles en la mejora de su proceso de enseñanza-aprendizaje (Figuras 6 y 7).

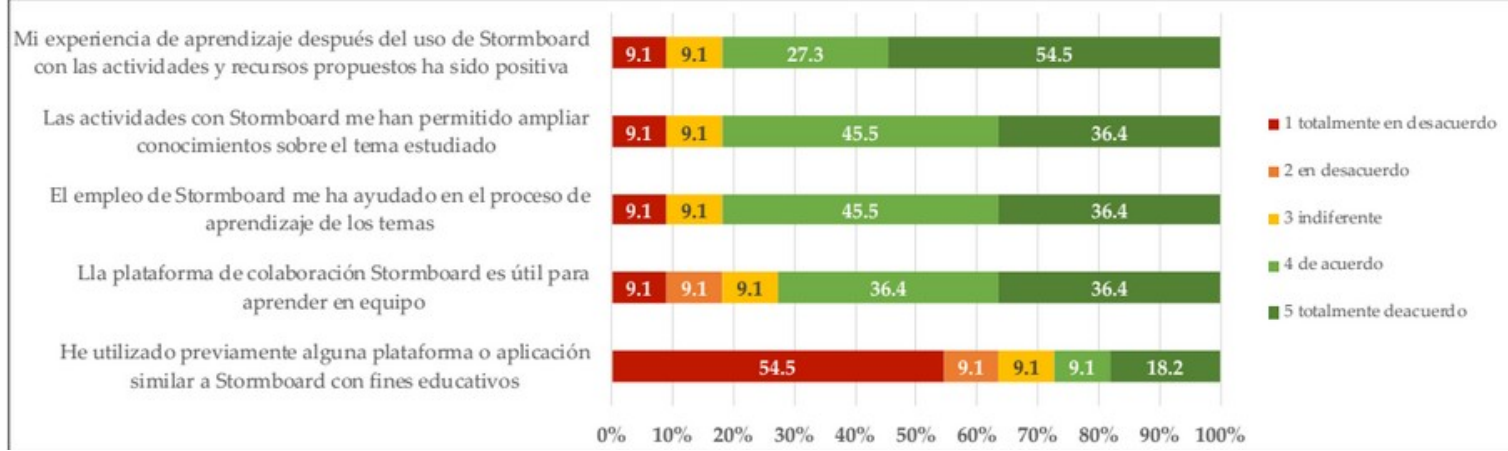

Figura 6. Valoración global del trabajo colaborativo con los tableros digitales (o storms) de Stormboard.

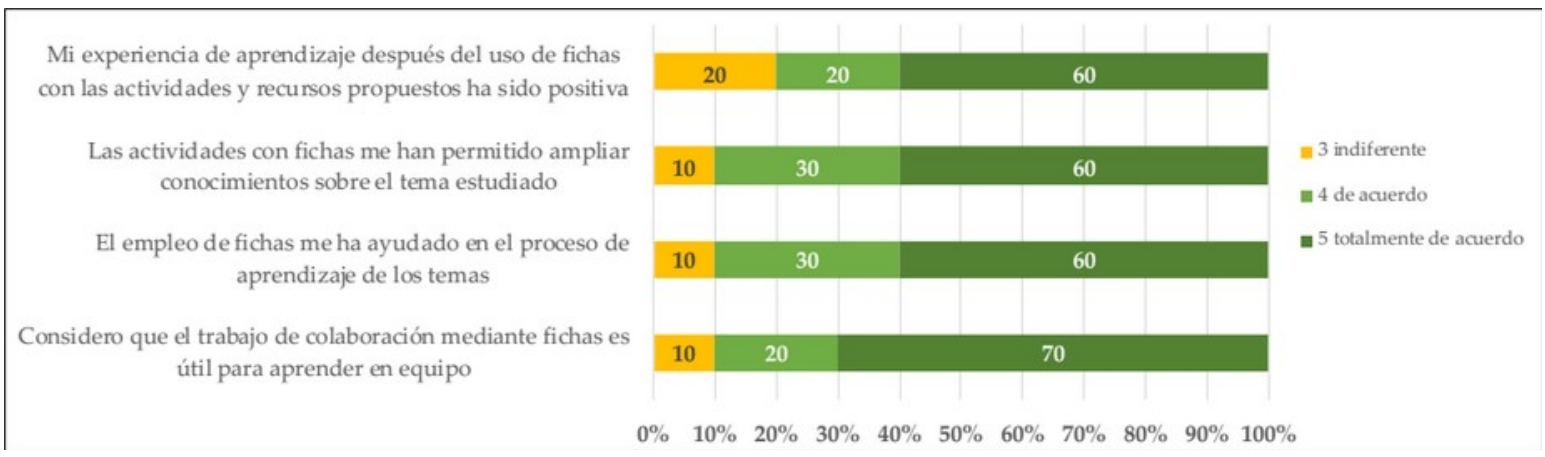

Figura 7. Grado de acuerdo del grupo control con la metodología docente tradicional (fichas y cartulinas).

\section{Discusión.}

La ES tiene la responsabilidad de formar profesionales competentes que sean capaces de aplicar y compartir sus conocimientos para el bien de las necesidades de 
la población que atienden, siendo necesario el correcto desempeño dentro de equipos de trabajo interdisciplinares presenciales o a distancia. Cada una de las habilidades que buscamos desarrollar en los futuros profesionales sanitarios debe entrenarse durante su formación con el fin de obtener los resultados deseados. Por un lado, estudios previos han demostrado que el AC promueve el desarrollo del pensamiento reflexivo, la adquisición de conocimientos de una forma más fácil y eficiente (más productivo en términos generales) y el aprendizaje humano, entre otros, en comparación con el aprendizaje individualista $(14,15)$. Por otro lado, la utilización de la simulación durante el curso académico ayuda a los profesionales de la salud a enfrentarse a la realidad laboral con más confianza y mayor preparación (16). Por todo ello, en el presente estudio se ha aplicado la lluvia de ideas como actividad colaborativa en el aula mediante el uso de la plataforma multimedia Stormboard, rompiendo así con el método de impartición clásica de las clases prácticas.

Las primeras observaciones apuntan a que nuestra experiencia didáctica, en la cual hemos usado la plataforma digital Stormboard como herramienta educativa para el desarrollo de lluvias de ideas como actividades de AC en el aula, ha resultado ser pionera tanto en la ES como en otras áreas de conocimiento de niveles educativos superiores, ya que, según la bibliografía revisada hasta la fecha, no existen registros al respecto. Esta plataforma colaborativa en la que se utiliza una pizarra online para crear lluvias de ideas surgió en el entorno empresarial, es decir, estaba dirigida principalmente a empresas interesadas en mejorar sus reuniones internas, la colaboración en línea y la planificación de un proyecto, por lo que es normal que no haya despertado mucho interés entre la comunidad educativa.

Aunque los mapas mentales se han utilizado durante mucho tiempo en el campo de la enseñanza con recursos convencionales (pizarra o fichas como en el caso del grupo control del presente estudio) y con otros programas como PowerPoint (17), el uso de aplicaciones móviles como Stormboard nos brinda mayores prestaciones que nos facilitan el control de participación del alumnado. Permite crear un entorno más cercano al productivo al añadirle el trabajo colaborativo, que favorece al desarrollo de habilidades sociales. Además, la versión digital del tradicional mural se constituye como una de las estrategias de aprendizaje más completas que podemos utilizar, trabajando simultáneamente la estructuración de contenidos y la fijación de nuevos conocimientos, y facilitando también el trabajo colaborativo, tan indispensable en la formación de nuestro alumnado (18). Por tanto, sugerimos más estudios que compartan la experiencia con el uso de esta plataforma multimedia para confirmar su utilidad en el proceso de la ES.

En cuanto al AC, nuestros resultados coinciden con otros estudios previos que sostienen que el trabajo colaborativo puede desarrollarse tanto presencialmente como asistido por ordenador (19-21). En líneas generales, nuestra experiencia didáctica ha tenido una gran acogida (81.8\%) entre el alumnado, a pesar de que el 63,6\% de los encuestados ha declarado no tener experiencia previa con el uso de la plataforma multimedia Stormboard. Ha quedado patente además que el desarrollo de las dos lluvias de ideas mediante dicha plataforma y las fichas (método tradicional) ha permitido crear nuevos espacios de AC en las clases prácticas, sean estos presenciales o virtuales $(22,23)$, en la cual la participación del alumnado ha sido alta y el entorno de trabajo saludable y competitivo como ha ocurrido en otro estudio previo centrado en la Eduación Secundaria Obligatoria (24). Asimismo, esta última observación está en línea con el alto grado de aceptación del alumnado con el aprendizaje móvil en el aula (o m-learning) $(25,26)$. El $80,1 \%$ de los encuestados ha manifestado sentirse muy 
de acuerdo o de acuerdo con este ítem, ya que piensan que les ha ayudado en su proceso de aprendizaje del temario.

No obstante, llama la atención que la satisfacción del estudiantado con la estrategia tradicional empleada (fichas) haya sido incluso mayor (90\%), permitiéndoles ampliar sus conocimientos sobre los contenidos impartidos. Este hecho pone de manifiesto que las lluvias de ideas en ambos entornos de aprendizaje (tradicional y digital), si están bien organizadas por el profesor y cumplen con los objetivos de la asignatura, facilitan la participación proactiva de los estudiantes en las clases y, por tanto, su motivación, contribución y satisfacción en las mismas también aumenta $(27,28)$. En esta misma línea, Betancur \& King (29) afirman que el diseño de mapas mentales, a mano o mediante el uso de software, representa una buena técnica para el pensamiento creativo y el aprendizaje, desarrollando así competencias fundamentales en el ámbito laboral, tales como el trabajo en equipo, integración de la información y las habilidades de comunicación.

Por otra parte, la aplicación de los cuestionarios gamificados con la aplicación móvil Socrative contribuyó a potenciar las observaciones anteriores. El mero hecho de salir de la monotonía del aula es de vital importancia para los alumnos, tal y como apuntan nuestros resultados y los de Parra-Santos et al. (30). Aunque no hemos encontrado diferencias significativas entre el grupo control y experimental, en ambos casos, son patentes los agradecimientos de los estudiantes acerca del uso de nuevas tecnologías con plataformas online como Socrative, que rompen la rutina de clase y les facilita participar activamente en la misma, permitiéndoles asimismo realizar las actividades con un mayor grado de interés y colaboración, influyendo así de manera considerable en su desempeño en clase (31). Nos parece llamativo que incluso aquellos participantes que mostraron menor interés por el módulo se divirtieran mientras respondían, aprendían y reforzaban sus conocimientos, obteniendo comentarios muy positivos al respecto en la encuesta (datos cualitativos no mostrados).

En relación con el impacto académico de nuestra experiencia innovadora en las notas del examen trimestral, es válido resaltar que existen autores que refieren resultados similares a los nuestros y otros que no. Así, por ejemplo, Martínez Pinilla et al. (32), en su estudio comparativo entre metodología tradicional vs. TIC, que llevó a cabo en las clases teóricas y prácticas de la asignatura Patología Celular en el Grado de Biología, manifiestan que, pese al interés de los alumnos por las nuevas metodologías educativas y el alto grado de implicación por parte de los profesores, el número de respuestas acertadas fue ligeramente mayor en aquellos estudiantes que completaron los cuestionarios gamificados, sin llegar a traducirse en una mejora sustancial del rendimiento académico. Estos hallazgos concuerdan con nuestros resultados.

Sin embargo, otras investigaciones recientes, como las de Martínez-Peinado et al. (33), si han observado diferencias significativas, de tal forma que el uso de Socrative ha favorecido la obtención de una calificación mayor en el examen final de la asignatura de Inmunología General. De los tres grupos en los que está dividida la asignatura, tan solo se aplicó Socrative en el grupo 1, a diferencia de nuestro estudio y el anteriormente citado. Otro dato relevante es el tamaño de su muestra, bastante mayor que el nuestro ( 75 frente a 25 estudiantes). Como es bien sabido, las muestras poblacionales pequeñas conllevan a una pérdida inherente de potencia en los contrastes de hipótesis, por tanto, es más difícil detectar diferencias significativas entre los grupos si realmente éstas existen y los resultados son más difíciles de 
interpretar. Esto último es, por consiguiente, lo que ha podido suceder en nuestro estudio.

Teniendo en cuenta todo lo anterior, se puede deducir que a pesar de que por el momento la implementación del AC y la evaluación de conocimientos adquiridos a través de herramientas gamificadas, como Stormboard y Socrative, en las aulas de Formación Profesional no constituyen una estrategia prioritaria ni preferida por los docentes, la vertiginosa dependencia que tiene la sociedad actual por el uso de herramientas tecnológicas, son motivo más que suficiente para que los docentes no ignoren esta realidad y las incorporen en sus aulas como recursos metodológicos innovadores para fomentar y estimular al alumno, logrando así una mayor atención y participación en su proceso de enseñanza-aprendizaje (34). Además, después de los últimos hechos acontecidos como consecuencia del estallido de la pandemia de enfermedad por Coronavirus 2019 (COVID-19), donde se ha constatado sobremanera que el profesorado se ha visto obligado a actualizarse a marcha forzada en un amplio abanico de herramientas digitales, cabe una profunda reflexión sobre el papel de la Educación y sus educadores en estos momentos con el fin de lograr una estrategia eficaz que mejore el rendimiento académico de los estudiantes Ciencias de la Salud.

Es evidente que la Transformación Digital en la Educación facilita una educación inmersiva en la que la experiencia de aprendizaje es más atractiva, inspiradora y transformadora, contribuyendo así a un aprendizaje más rápido, eficiente y colaborativo. Por consiguiente, dado que parece innegable que estamos experimentando los primeros efectos del cambio educativo, en el que convergen los espacios presenciales y virtuales (entornos híbridos), parece razonable pensar que estar al día en el uso de las nuevas tecnologías es vital para mejorar nuestra actuación docente en los distintos contextos formativos. La gamificación educativa digital será una herramienta fundamental en el futuro próximo, favoreciendo innovaciones en el aula, más allá de los experimentos novedosos. No obstante, el principal desafío para el sistema educativo español actual es romper la brecha digital de su profesorado. Es requisito indispensable que este se transforme, cuanto antes, en los llamados tecnófilos innovadores $(35,36)$, para que pueda darse tal cambio de paradigma.

\section{Conclusiones.}

- La lluvia de ideas representa un excelente recurso educativo colaborativo que motiva notablemente a los estudiantes del módulo profesional de Técnicas de Ayuda Odontológica/Estomatológica y les permite interactuar entre iguales y con los docentes, saliendo de esta manera de su zona de confort e impulsando el aprendizaje significativo, independientemente de que el entorno colaborativo sea tradicional o virtual.

- Stormboard es una herramienta digital innovadora prácticamente desconocida en el ámbito educativo que permite el desarrollo de actitudes y comportamientos colaborativos, así como el aprendizaje en los contextos donde los alumnos más tarde van a trabajar como profesionales. Se podría aplicar a otros Ciclos Formativos o niveles educativos superiores como el universitario con las adaptaciones pertinentes.

- Los resultados evidenciados en esta experiencia didáctica innovadora demuestran la necesidad de introducir cambios tanto en las metodologías de enseñanza-aprendizaje como en la variedad de estrategias de evaluación a través de plataformas multimedia como Socrative.

- La gamificación educativa con Socrative es una actividad inmersiva que mejora notablemente la motivación de los alumnos y fomenta el estudio autónomo de 
una forma más divertida, atractiva y acorde al perfil socioeducativo del alumnado actual.

Material suplementario: No se aporta material suplementario.

Financiación: El presente trabajo no contó con financiación.

Agradecimientos: A la profesora Dña. Encarna Abenza Mañas del I.E.S. Los Albares (Cieza, Murcia) por su colaboración e incondicional apoyo durante el transcurso de las prácticas tuteladas y desarrollo de este estudio.

Autorías: Todos los autores han contribuido en el diseño, la elaboración y la revisión del trabajo.

Declaración de conflicto de interés: Los autores declaran no tener ningún conflicto de intereses.

\section{Referencias}

1. Moreno-Jimenez B, Blanco-Donoso L, Aguirre Camacho A, de Rivas S, Herrero M. Habilidades Sociales para las Nuevas Organizaciones. Psicol. conduct, 2014, 22, 585-602. En https://www.behavioralpsycho.com/wp-content/uploads/2019/08/11.Moreno 22-3oa.pdf (visitado en 5 May 2020).

2. Mumford MD, Peterson NG, Childs RA. Basic and cross-functional skills. En An occupational information system for the 21st century: The development of $O^{*} N E T$; $1^{\text {a }}$ ed.; NG Peterson, MD Mumford, WC Borman, PR Jeanneret, EA Fleishman, Eds.; Editorial: APA PsycBooks, Washington, USA, 1999, 49-69. https://doi.org/10.1037/10313-000 (visitado en 7 Abr 2020).

3. Bolaño C. Sociedad de la Información, reestructuración productiva y economía del conocimiento. Telos, 2018, 64.2 Disponible en: https:/telos.fundaciontelefonica.com/archivo/numero064/sociedad-de-la-informacionreestructuracion-productiva-y-economia-del-conocimiento/ (visitado en 5 Jun 2020).

4. Cortés MMD. Comunicación enfermera/paciente: Reflexión sobre la relación de ayuda. Rev Esp Com Sal, 2012, 2, 55-61. Disponible en: https://e-revistas.uc3m.es/index.php/RECS/article/view/3405/2056 (visitado en 4 Mar 2020).

5. Blanco AV, Amigo JC. El rol del docente en la era digital. RIFOP, 2016, 30, 103-114. Disponible en: http://www.redalyc.org/articulo.oa?id=27447325008 (visitado en 1 Feb 2020).

6. Manrique CRC, Puente RMT. El constructivismo y sus implicancias en educación. Educación, 1999, 217-244. Disponible en: https://dialnet.unirioja.es/servlet/articulo? codigo $=5056798$ (visitado en 1 Feb 2020).

7. Colorado-Aguilar BL, Edel-Navarro R. La usabilidad de TIC en la práctica educativa. RED, 2012, 30, 1-11. Disponible en: https://www.um.es/ead/red/30/edel.pdf (visitado en 9 Jun 2020).

8. Bodero L, Alvarado Z. Los beneficios de aplicar las TICs en la Universidad. Yachana Revista Científica, 2014, 3, 119-125. Disponible en: http://revistas.ulvr.edu.ec/index.php/yachana/article/view/23/18 (visitado en 9 Jun 2020).

9. Onrubia J. Aprender y enseñar en entornos virtuales: Actividad conjunta, ayuda pedagógica y construcción del conocimiento. RED, 2016, 50, 1-14. Disponible en: https://revistas.um.es/red/article/view/270801 (visitado en 5 May 2020).

10. Henao Martínez D, Vázquez Navarrete ML, Vargas Lorenzo I. Factores que influyen en la coordinación entre niveles asistenciales según la opinión de directivos y profesionales sanitarios. Gac Sanit, 2009, 23, 280-286. https://doi.org/10.1016/j.gaceta.2008.05.001 (visitado en 5 May 2020).

11. Morillo JM y Pajares Y. Técnicas de Ayuda Odontológica y Estomatológica GM. Libro alumno. $1^{a}$ ed.; Editorial: McGraw-Hill Interamericana, Madrid, España, 2017. 
12. Modelo de evaluación de la actividad docente del profesorado de la UCM: programa DOCENTIA-UCM. 2016. Disponible online: https:/www.ucm.es/data/cont/docs/3-2016-1115-Docentia-noviembre2016.pdf (visitado en 21 Sep 2020).

13. Boletín Oficial del Estado. Real Decreto 463/2020, de 14 de marzo, por el que se declara el estado de alarma para la gestión de la situación de crisis sanitaria ocasionada por la COVID-19. Disponible online: https://www.boe.es/buscar/doc.php?id=BOE-A-2020-3692 (visitado en 25 Abr 2020).

14. Maldonado Pérez MI. El trabajo colaborativo en el aula universitaria. Laurus, 2007, 13, 263-278. Disponible en: https://www.redalyc.org/pdf/761/76102314.pdf (visitado en 2 Abr 2020).

15. Millis BJ. Cooperative Learning. En Materials presented at The University of Tennessee at Chattanooga Instructional Excellence Retreat. Human-Computer Interaction Series. Ablex Publishing Corporation, Norwood, New Jersey, USA, 1996. Disponible en: http://www.utc.edu/Teaching-Resource-Center/CoopLear.htm (visitado en 1 Feb 2020).

16. Muro Sans JA. Hacia nuevos modelos de enseñanza-aprendizaje en ciencias de la salud. En Educación Médica, 91-99, Jornada de la Sociedad de Educación Médica de Euskadi (SEMDE), Bilbao, España, 2010. Disponible en: http://scielo.isciii.es/scielo.php? script=sci_arttext\&pid=S1575-18132011000200004 (visitado en 8 Mar 2020).

17. Muñoz González JM, Serrano Rodríguez R, \& Marín Díaz V. El aprendizaje colaborativo y su desarrollo a través de mapas mentales. Una innovación educativa en la formación inicial docente. Educatio Siglo XXI, 2014, 32, 193-212. https://doi.org/10.6018/j/194151 (visitado en 3 Mar 2020).

18. Redondo AC, Luque AG. Los murales digitales para un aprendizaje cooperativo de la Historia: Una herramienta innovadora. Ensayos, Revista de la Facultad de Educación de Albacete, 2018, 33, 113-127. https://doi.org/10.18239/ensayos.v33i1.1745 (visitado en 12 May 2020).

19. González Burgos E, Serrano López DR. Diseño y uso de materiales para la docencia presencial y virtual. En IV Jornadas de TIC e Innovación en el Aula, 1-7, La Plata, Argentina 2017.

Disponible

en:

http://sedici.unlp.edu.ar/bitstream/handle/10915/65483/Documento_completo_.pdf-

PDFA.pdf?sequence $=1$ \&isAllowed $=\mathrm{y}$

20. González González C. Gamificación en el aula: ludificando espacios de enseñanzaaprendizaje presenciales y espacios virtuales. 2019. Disponible en: https://doi.org/10.13140/RG.2.2.34658.07364_(visitado en 10 Oct 2020).

21. González Burgos E, Serrano López DR. Diseño y uso de materiales para la docencia presencial y virtual. En IV Jornadas de TIC e Innovación en el Aula, 1-7, La Plata, Argentina, 2017. Disponible en: http://sedici.unlp.edu.ar/handle/10915/65483 (visitado en 1 Oct 2020).

22. Alfageme González MB. Modelo colaborativo de enseñanza-aprendizaje en situaciones no presenciales. Un estudio de caso. Tesis doctoral. 2003. Disponible https://dialnet.unirioja.es/servlet/tesis?codigo=18830 (visitado en 2 May 2020).

23. González RMG, González LG, Cruz NM, Fuentes MGL, Aguirre EIR, González EV. Acercamiento epistemológico a la teoría del aprendizaje colaborativo. Apertura, 2012, 4, 156-169. En: http://www.udgvirtual.udg.mx/apertura/index.php/apertura/article/view/ 325/290 (visitado en 3 May 2020).

24. Jiménez Rodríguez MA. Las TIC en las prácticas de evaluación en Educación Secundaria Obligatoria. Espiral, Cuadernos del profesorado, 2016, 9, 56-67. Disponible en: http://espiral.cepcuevasolula.es/ (visitado en 2 Feb 2020).

25. Fombona Cadavieco J, Rodil Pérez FJ. Niveles de uso y aceptación de los dispositivos móviles en el aula. Píxel-Bit. Revista de Medios y Educación, 2018, 52, 21-35. https://doi.org/10.12795/pixelbit.2018.i52.02 (visitado en 1 Abr 2020). 
26. López-Hernández FA, Silva-Pérez MM. Factores que inciden en la aceptación de los dispositivos móviles para el aprendizaje en educación superior. Estudios sobre Educación, 2016, 30, 175-195. https://doi.org/10.15581/004.30.175-195 (visitado en 25 Abr 2020).

27. Álvarez-Álvarez MB. Adaptación del método docente al Espacio Europeo de Educación Superior: La motivación de los alumnos como instrumento clave. Estudios sobre Educación, 2005, 9, 107-126. https://dadun.unav.edu/handle/10171/8911 (visitado en 12 May 2020).

28. Álvarez C, Silió G, Fernández E. Planificación, colaboración, innovación: tres claves para conseguir una buena práctica docente universitaria. REDU, 2012, 10, 415-430. https://doi.org/10.4995/redu.2012.6138 (visitado en 6 May 2020).

29. Betancur MIG, King G. Using mind mapping as a method to help ESL/EFL students connect vocabulary and concepts in different contexts. Revista Trilogía, 2014, 6, 69-85. Disponible en: https://dialnet.unirioja.es/servlet/articulo?codigo=4785353 (visitado en 1 May 2020).

30. Parra-Santos T, Molina JM, Milanovic I, Casanova G, Luna G. SOCRATIVE como Herramienta de Diagnóstico y Autoevaluación del Proceso de Aprendizaje. En Del verbo al bit, 2 ${ }^{\text {a }}$ ed.; Herrero FJ, Mateos C, Toledano S, Ardèvol A, Trenta M Eds.; Editorial: Sociedad Latina de Comunicación Social, La Laguna, España, 2017, 130-139. Disponible en: http://www.revistalatinacs.org/16SLCS/2017 libro/006 Parra.pdf (visitado en 1 May 2020).

31. Frías MV, Arce C, Flores-Morales P. Uso de la plataforma «socrative.com» para alumnos de Química General. Educ Química, 2016, 27, 59-66. http://dx.doi.org/10.1016/j.eq.2015.09.003 (visitado 1 Abr 2020).

32. Martínez Pinilla E, Vega Naredo I, Navarro Incio A, Bermejo Millo JC. Uso de la plataforma Socrative en las clases expositivas: como sacar partido a los dispositivos móviles en el aula. Universidad de Oviedo, 2018, 1-22. Disponible en: https://docplayer.es/174731068-Uso-dela-plataforma-socrative-en-las-clases-expositivas-como-sacar-partido-a-los-dispositivosmoviles-en-el-aula.html (visitado 3 Oct 2020).

33. Martínez-Peinado P, Pascual-García S, López Jaén AB, Cobo R, Peiró G, Navarro Blasco FJ, Sempere Ortells JM. Aprendizaje de la Inmunología a través de casos clínicos mediante el uso de Socrative ${ }^{\circledR}$. Memorias del Programa de Redes-I3CE de calidad, innovación e investigación en docencia universitaria, 2019, 2461-2475. Disponible en: https://rua.ua.es/dspace/bitstream/10045/102029/1/Memories-Xarxes-I3CE-2018-19181.pdf (visitado en 27 Feb 2020).

34. Benítez-Porres J. Socrative como herramienta para la integración de contenidos en la asignatura "Didáctica de los Deportes". En XXII Jornadas Internacionales de Innovación Universitaria "Educar para transformar", 824-831, Madrid, España, 2014. Disponible en: http://hdl.handle.net/11268/4513 (visitado en 6 Jul 2020).

35. Arancibia ML, Valdivia I, Araneda SM, Cabero-Almenara J. Tipologías para la Innovación tecnológica en Docentes de Educación Superior a partir de un análisis de conglomerados: un estudio exploratorio. RED, 2017, 55, 1-21. https://doi.org/10.6018/red/55/5 (visitado en 5 Jun 2020).

36. Pérez Sánchez B, Salas Madrid F. Hallazgos En Investigación Sobre El Profesorado Universitario Y La Integración De Las Tic En La Enseñanza. Revista Electrónica «Actualidades Investigativas en Educación», 2009, 9, 1-25. Disponible en: https://www.redalyc.org/pdf/447/44713054006.pdf (visitado en 3 Jun 2020).

(C) 2020 por los autores. Enviado para su publicación en acceso abierto bajo los términos y condiciones de la licencia Creative Commons Attribution (CC BY) (http://creativecommons.org/licenses/by/4.0/). 